\title{
A new large high latitude cone-like far-IR nebula
}

\author{
B. Aryal and R. Weinberger
}

Institut für Astrophysik, Universität Innsbruck, Technikerstraße 25, 6020 Innsbruck

e-mail: [Binil. Aryal; Ronald. Weinberger]@uibk.ac.at

Received 26 November 2004 / Accepted 4 October 2005

\section{ABSTRACT}

We present a new isolated interstellar nebula $\left(\mathrm{RA}=08^{\mathrm{h}} 27^{\mathrm{m}}\right.$, Dec $\left.=+25^{\circ} 54^{\prime}(\mathrm{J} 2000) ; \ell=197^{\circ} .8, b=+31^{\circ} .6\right)$ found at $100 \mu \mathrm{m}$ and $60 \mu \mathrm{m}$ on IRAS maps. It has dimensions of $\sim 140^{\prime} \times 70^{\prime}$ and a cone-like shape, suggesting interaction with ambient matter or external radiation. The nebula contains 2 bright condensations ("nuclei") and several prominent filaments, the latter being approximately parallel to each other. We carried out preliminary studies based on IRAS data and the Palomar Observatory Sky Survey and found that there are no hints of star formation in the nebula. The nebula's long axis is almost parallel to the Galactic plane. A shaping due to the nebula's motion through the interstellar medium might be not a sufficient reason in this case. We have discussed this possibility by applying results of Reynolds number hydrodynamics. During a search for possible stellar candidates for shaping this nebula we noted a remarkable position of one of the very few nearby $(D \approx 0.36 \mathrm{kpc})$ pulsars known at about this latitude: PSR B0823+26. This pulsar is projected at the tip of the nebula and its proper motion is approaching along the long axis $\left(\sim 180^{\circ}\right.$ to the direction of the cone) of the nebula. The true cause for the shaping of the nebula, which was coined Skeleton Nebula by us, however remains unknown.

Key words. infrared: ISM - ISM: structure

\section{Introduction}

Comet-like or cone-like morphologies of (large) interstellar nebulae are usually considered to be clear hints of interaction processes. The number of objects of this type is rather small, as was demonstrated by Odenwald \& Rickard (1987) and Odenwald (1988), who had performed a visual survey of all IRAS sky maps in the $100 \mu \mathrm{m}$ bandpass. They discovered 15 high Galactic latitude clouds with comet-like appearance and discussed their far-IR and optical properties. It was shown that the shapes of some of these clouds could be explained via their motion through the interstellar medium. In order to enlarge this sample and to find possible other shaping mechanisms, any additional detection of nebulae of this morphological type may be of interest.

In this paper, we present a large new high latitude conelike cloud found by us at $100 \mu \mathrm{m}$ and $60 \mu \mathrm{m}$ IRAS images. A description of this nebula as well as some physical properties are given in Sects. 2 and 3. A discussion of some stellar objects that might shape the nebula is presented in Sect. 4. Finally, a brief hydrodynamic discussion and our conclusions are given in Sects. 5 and 6, respectively.

\section{Observations}

Figures $1 \mathrm{a}, \mathrm{b}$ show $4.5^{\circ} \times 4.5^{\circ}$ IRAS 100 and $60 \mu \mathrm{m}$ images centered at RA $=08^{\mathrm{h}} 27^{\mathrm{m}} 05^{\mathrm{s}}$, Dec $=+25^{\circ} 53^{\prime} 59^{\prime \prime}(\mathrm{J} 2000)$, i.e. at $\ell=197.79, b=+31^{\circ} .58$. A remarkable A-shaped nebula with a skeleton-like morphology (hence dubbed as "Skeleton Nebula" by us, hereafter $\mathrm{SkN}$ ) with a size of $\sim 2.5$ can be seen. In the optical, very faint and diffuse emission is visible on a Digital Sky Survey (DSS) image (Fig. 1c).

\subsection{Star counts}

The two nuclei (black extended regions in Figs. 1a,b and + signs in Figs. 1c,d,e) might be dense portions of the nebula containing embedded young stars, i.e. the whole object might be a (nearby) star forming region. To check this possibility, we have carried out star counts in order to compare the stellar density at the location of the nuclei with the stellar densities of locations outside. In case of star formation we would expect noticeable dust extinction in these nuclei, i.e. less stars due to the obscuration or dimming of background stars or perhaps a concentration of newly born stars within the nuclei. The counts were done visually using a microscope $(25 \times$ magnification $)$ on a Second Palomar Observatory Sky Survey blue (POSS II B) film copy. Each grid size was $15^{\prime} \times 15^{\prime}$ (i.e. an area comparable with that of each of the two nuclei). A total of 17 grids were chosen for the star counts: one each at the location of the nuclei, 5 at the filaments of the SkN, and 10 outside of the nebula. No stellar concentration was found within the nuclei.

The average number of stars counted at the locations of the nuclei, of the filaments and in the regions around the SkN were 107, 109, and 103 respectively. The observational and statistical errors were $\pm 5 \%$ and $\sim \pm 9 \%$, respectively. Hence, 

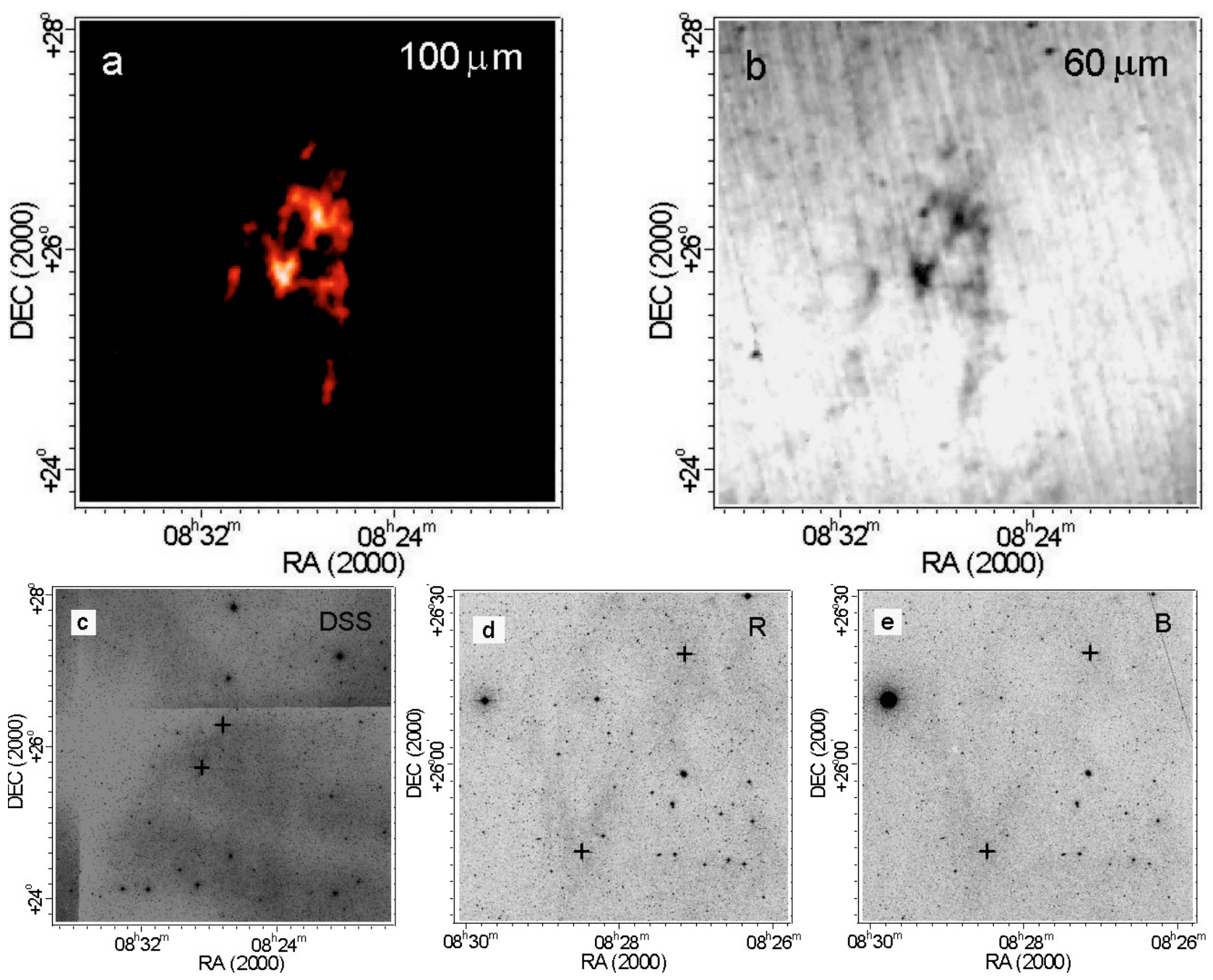

Fig. 1. A $4.5^{\circ} \times 4.5^{\circ}$ IRAS image at $100 \mu \mathrm{m}$ a) and $60 \mu \mathrm{m} \mathrm{b}$ ) of the "Skeleton Nebula". The image is centered at RA $=08^{\mathrm{h}} 27^{\mathrm{m}} 05^{\mathrm{s}}$, Dec $=$ $+25^{\circ} 53^{\prime} 59^{\prime \prime}$ (J2000). c) A DSS image (red-sensitive POSS I) of the same field. d) A POSS II R and e) a POSS II B field showing the central region of Fig. 1b. In the panels c), d), and e) the plus (+) signs mark the positions of the two main condensations ("nuclei") of the nebula as seen in Fig. 1a.

the difference is well within the error limit. In other words, the stellar density is the same at the locations of the nuclei and at the other positions within and outside of the nebula. This (small) difference also remains within the $\pm 5 \%$ error limit when the counts were repeated by increasing and then decreasing the grid size by $25 \%$. These results suggest that dust extinction is not high in the optical, since it does not influence the number of visible background stars. Hence we found that the SkN probably does not represent a star forming region.

The central region of the $\mathrm{SkN}$ including the nuclei was examined using R and B film copies from POSS II, (Figs. 1d,e): there, very faint diffuse extended structures having nearly constant optical surface brightness $\left(\sim 24 \mathrm{mag} / \operatorname{arcsec}^{2}\right)$ are present at and around the position of these nuclei. The heart-like morphology of the southern nucleus found at $100 \mu \mathrm{m}$ and $60 \mu \mathrm{m}$ can be seen in POSS II R too, and has about the same size there. Taken as a whole, the Skeleton Nebula is hardly brighter in $\mathrm{R}$ compared to $\mathrm{B}$. This suggests a reflection nebula, i.e. a nebula containing some dust, but a certain contribution of faint optical emission lines in the red wavelength range cannot be ruled out.

\subsection{Categorizing the nebula}

The nebula belongs to the "Category II cloud" of Odenwald (1988). Odenwald carried out optical and far-IR studies on 14 high Galactic latitude clouds with comet-like or filamentary appearances. Category II clouds, appearing as faint, diffuse reflection nebulae of nearly constant optical surface brightness but showing a clumpy internal structure, comprise the objects G64-26, G208-28, G225-66, G228-27, G239-15 as well as the Draco cloud, G90+38. Odenwald (1988) estimates that category II clouds have relatively low masses in the range 3-40 $M_{\odot}$ and surface brightness typical for clouds illuminated by the general interstellar radiation field.

The SkN's two bright nuclei are located at RA = $08^{\mathrm{h}} 28^{\mathrm{m}} 30^{\mathrm{s}}$, Dec $=+25^{\circ} 43^{\prime} 58^{\prime \prime}(\mathrm{J} 2000)$ and RA $=08^{\mathrm{h}} 27^{\mathrm{m}} 06^{\mathrm{s}}$, Dec $=+26^{\circ} 20^{\prime} 10^{\prime \prime}(\mathrm{J} 2000)$, respectively. The angular separation between the nuclei is $\sim 39^{\prime}$. At $100 \mu \mathrm{m}$ (Fig. 1a), the northern nucleus $\left(\mathrm{N}_{1}\right)$ displays 3 bright, short $\left(0^{\circ} 25\right)$, "arms" apparently originating in its brightest, that is central, region. One extends towards the north-east, the second one towards the 


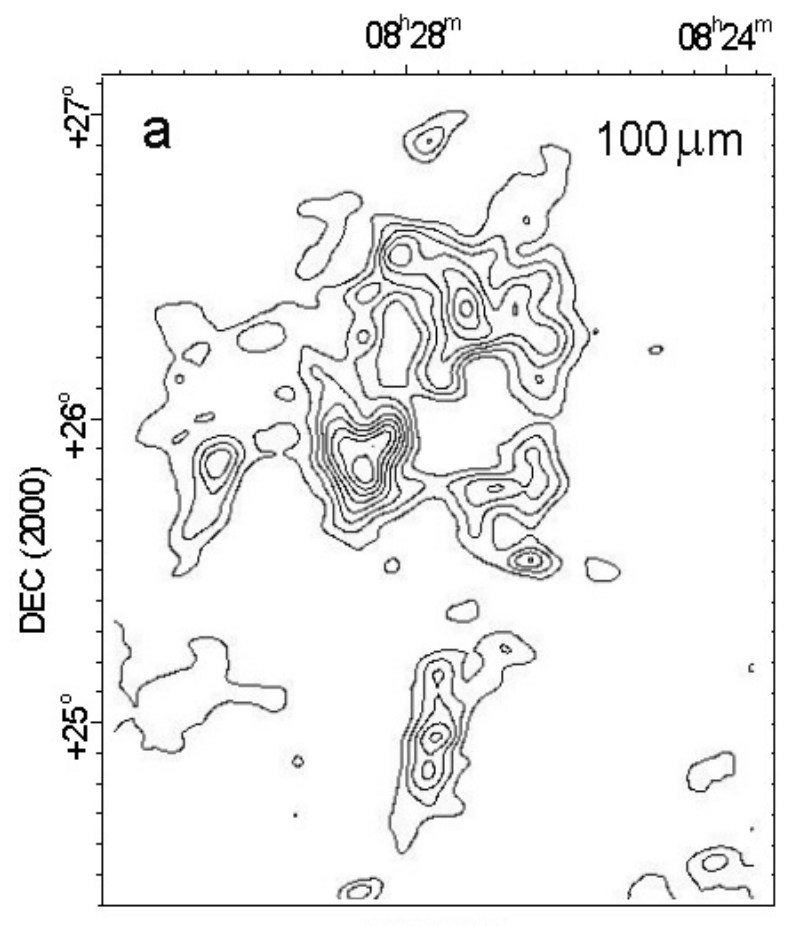

$\mathrm{RA}(2000)$

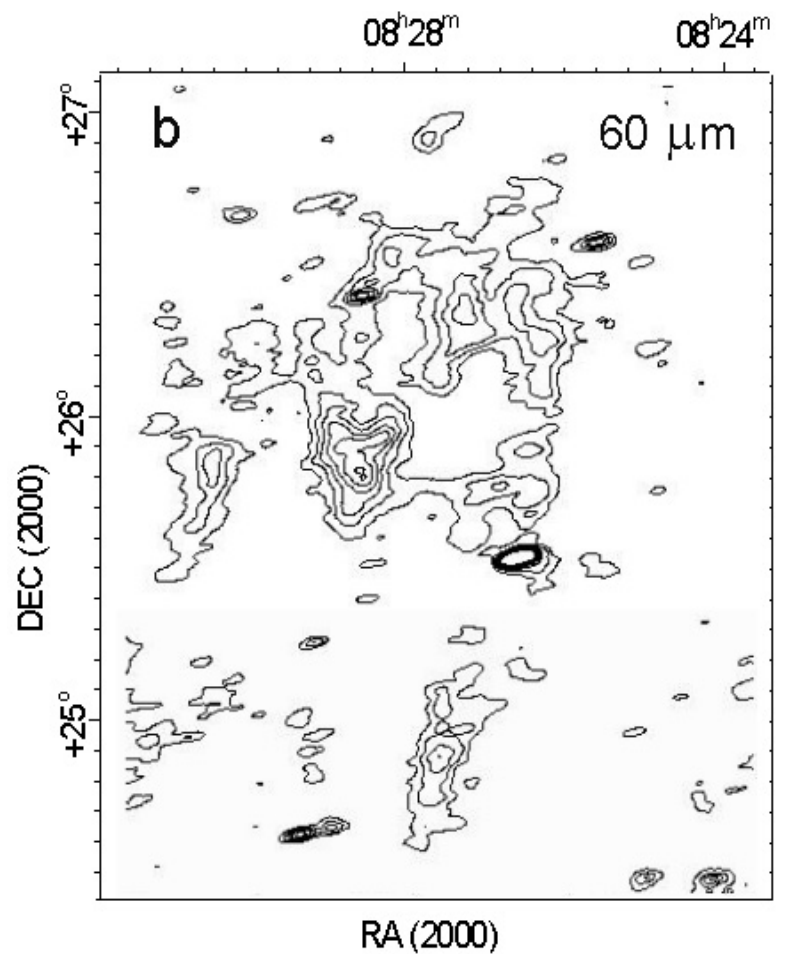

Fig. 2. $100 \mu \mathrm{m}$ a) and $60 \mu \mathrm{m}$ b) HIRES contour maps of the Skeleton Nebula. The IRAS (HIRES) contours span 0.55 to $4.05 \mathrm{MJy} / \mathrm{str}$ (for $100 \mu \mathrm{m}$ ) and 0.15 to $1.95 \mathrm{MJy} / \mathrm{str}$ (for $60 \mu \mathrm{m}$ ) in steps separated by 0.50 and 0.20 , respectively.

south-west, and the third is perpendicular, extending towards the south-east. Both eastern and western arms further sharply (by $\sim 90^{\circ}$ ) bend southwards. Together with the above perpendicular arm all these extensions look like an upside down small letter $\omega$.

The southern nucleus $\left(\mathrm{N}_{2}\right)$ bears some resemblance to a scaled up version of $\mathrm{N}_{1}$ and its arms (Figs. 1a,b). The longest arm (in the west) extends from north to south. The apparent size of this filament is $\sim 80^{\prime}$. It should be noted that all arms, i.e. all filamentary structures associated with both nuclei are approximately parallel (within $\pm 15^{\circ}$ ) to each other and extend towards south or south-east. Hence, the remarkable structure of the SkN indicates that some external force is influencing its shape. The source of this force seems to be located in the north-west of the nebula.

The SkN is not only discernible at $100 \mu \mathrm{m}$, but also at $60 \mu \mathrm{m}$. More details than in Fig. 1 can be seen in Fig. 2, which displays $100 \mu \mathrm{m}$ and $60 \mu \mathrm{m}$ HIRES contour maps (resolution $\sim 1^{\prime}$ ). In Figs. 2a,b one notices that i) both nuclei are connected to the filamentary structures mentioned above; ii) these filamentary structures deviate towards south; iii) these structures are approximately parallel to each other; iv) there is a bridge-like connection between both nuclei; v) the western filamentary structures are much more extended than the eastern ones; and vi) knots can be seen in the western filament and in the northern region.

\subsection{Inclination}

Although it is possible that the SkN's long axis is in the sky plane, it is more probable that it is inclined by a certain angle with respect to the plane of the sky. Possibly, $\mathrm{N}_{1}$ and $\mathrm{N}_{2}$ might, seen from the location of an assumed shaping stellar source in the north-east, be at the same distance from the source. If so, how strongly must the SkN be inclined? The inclination angle $i$ (angle between the line of sight and the normal vector of the plane of the nebula) can be estimated using $\cos ^{2} i=$ $\left[(b / a)^{2}-0.2^{2}\right] /\left(1-0.2^{2}\right)$ with $b / a$ as the measured axial ratio (Holmberg 1946). The inclination angle $(i)$ is found to be $\sim 54^{\circ}$. Thus, our nebula is neither face-on $\left(i \rightarrow 0^{\circ}\right)$ nor edge-on $(i \rightarrow$ $90^{\circ}$ ) object. The $\mathrm{SkN}$ will be edge-on (minor axis/major axis $\sim 0.20, i=90^{\circ}$ ) if we rotate it by $\sim 36^{\circ}$ (i.e. $90^{\circ}-54^{\circ}$ ) from the southern to the northern direction (assuming that the nebula is in the sky plane). At this angle, both nuclei would lie in a plane and any discrete object which would be located at the head of the nebula in the north-east would almost lie above in between the nuclei. In such a case the true size of the skeleton nebula would be larger by a factor of about $2.5 \times$ the apparent size.

\section{Physical properties}

\subsection{Counterparts at various wavelengths?}

There is a rapid decrease of the nebula's surface brightness towards shorter infrared wavelengths as is evident by comparing the nebula at $100 \mu \mathrm{m}, 60 \mu \mathrm{m}, 25 \mu \mathrm{m}$ and $12 \mu \mathrm{m}$. All traces of the filamentary structures as well as both nuclei have vanished at $25 \mu \mathrm{m}$, whereas a very faint remnant of $\mathrm{N}_{2}$ remains visible at $12 \mu \mathrm{m}$. We have obtained far-IR surface brightness measurements at each of the four passbands for the nebular region using IRAS images from Groningen IRAS server (SRON, Groningen). The colour correction depends on the shape of the 
intrinsic energy distribution and on the details of the wavelength response of the system. Corrections for a number of input energy distributions are given in Table 6, Chap. 6 of the "IRAS Explanatory Supplement" (1985). The ratio of flux densities before the colour correction corresponds to $240 \mathrm{~K}$ of intrinsic temperature. The correction factor corresponding to this temperature as given in Table 6 is used for the colour correction.

A comparison of far-IR spectral distributions of our nebula with the Draco cloud (Odenwald \& Rickard 1987) and the nebula MBM 20 (Weiland et al. 1986) is shown in Fig. 3. The SkN (solid line in Fig. 3) does not have a strong $12 \mu \mathrm{m}$ and $25 \mu \mathrm{m}$ spectral component. This lack of emission at $12 \mu \mathrm{m}$ and $25 \mu \mathrm{m}$ suggests that the dust population may be relatively devoid of a small grain component as compared to the cirrus material (Puget et al. 1985), or perhaps that the small dust grain are not being adequately excited by the ambient radiation field (Mebold et al. 1985). The strength of the stellar radiation field near the Draco cloud is estimated to be about $10 \times$ lower than in the Galactic plane (Mebold et al. 1985). The Draco cloud is at $38^{\circ}$ latitude, at slightly higher latitude than the $\mathrm{SkN}\left(32^{\circ}\right)$. We estimate that the stellar radiation field for the $\mathrm{SkN}$ is also weaker $(\sim 10-12 \times)$ with respect to a position in the Galactic plane not only because of its high latitude but also due to the smaller number of stars in this region due to the longitude of $\ell=198^{\circ}$.

We used SkyView (http://skyview.gsfc.nasa.gov/) in order to search for counterparts of the $\mathrm{SkN}$ at $\mathrm{H} \alpha$ using the all-sky $\mathrm{H} \alpha$ map, a composite of the Virginia Tech Spectral line Survey (VTSS) in the north and the Southern H-Alpha Sky Survey Atlas (SHASSA) in the south, and in X-rays (ROSAT All Sky Survey (RASS3), RXTE/PCA All Sky Slew Survey (XSS)), as well as at radio wavelengths (Sydney University Molonglo Sky Survey (SUMSS), the VLA FIRST Survey, and the NRAO VLA Sky Survey (NVSS)), but no emission could be detected in any of these.

\subsection{Temperature, distance, and mass estimate}

We have determined the dust colour temperature $\left(T_{\mathrm{d}}\right)$ of the nebula using Henning et al. (1990). The average dust colour temperature in the region was determined to be $27 \pm 4 \mathrm{~K}$. We also determined the dust temperature distribution throughout the nebular region by dividing the $100 \mu \mathrm{m}$ map by the $60 \mu \mathrm{m}$ map and comparing the resulting $I(100 \mu \mathrm{m}) / I(60 \mu \mathrm{m})$ values at each map location with the values given for dust grain models by Dwek (1986). We found that the dust colour temperature of the southern nucleus is $34 \pm 4 \mathrm{~K}$ and the temperature of its northern counterpart $32 \pm 4 \mathrm{~K}$. The eastern filamentary structures are slightly cooler $(20 \pm 2 \mathrm{~K})$ than the western filamentary structures $(26 \pm 3 \mathrm{~K})$. Hence, the locations of the maximum emission i.e. the nuclei of the $\mathrm{SkN}$ are warmer than their filaments.

The distance of the nebula is unknown. In our case, the star count method (Mebold et al. 1985) does not help because of insufficient extinction and too few stars in the region. The nebula also has no obviously embedded stars, which would be

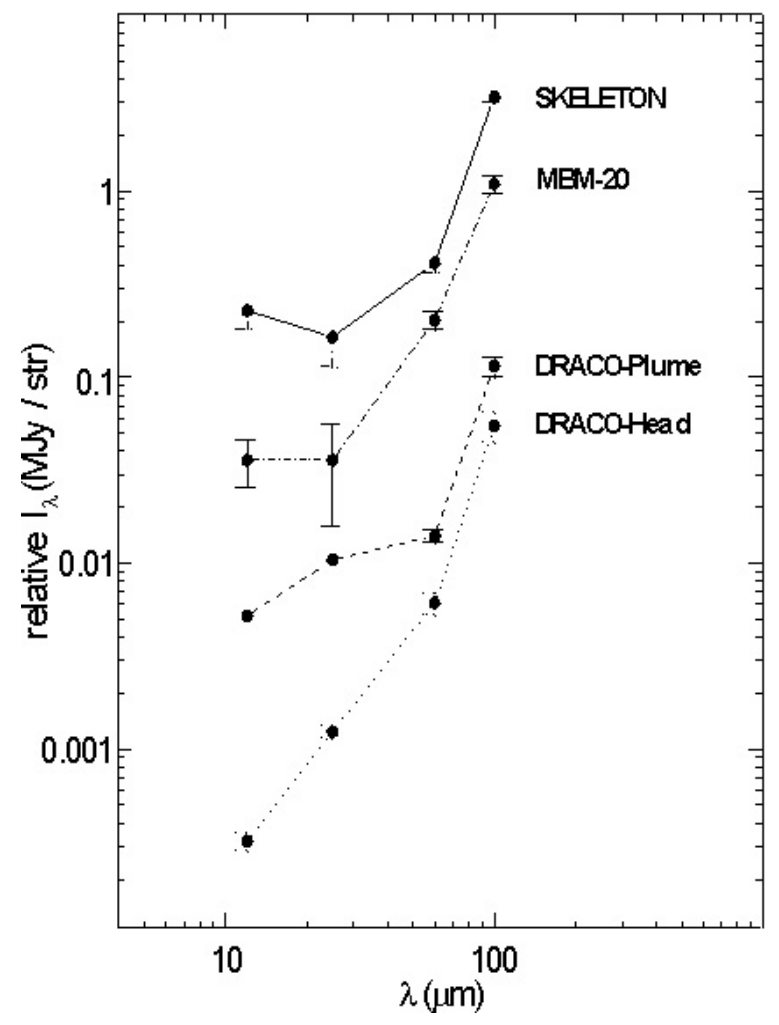

Fig. 3. Far-IR spectral distributions of the Skeleton Nebula (solid line). The major cloud component (DRACO-Head) and a segment of the plume (DRACO-Plume) of the Draco cloud are represented by dotted and dashed lines, respectively (Odenwald \& Rickard 1987). A dotdashed line is used for the MBM 20 "cirrus cloud" (position No. 1 from Table 1, Weiland et al. 1986). All intensities have been background subtracted and colour corrected for $240 \mathrm{~K}$ blackbody emission. The spectra have been rescaled by dividing the intensities for the Skeleton Nebula, Draco head, Draco plume, and MBM 20 by 5.5, 45.6, 9.5, and 14.0, respectively.

helpful for this purpose. Following Odenwald (1988), a distance of $200 \mathrm{pc}$ will be assumed for the moment. The maximum physical size of the nebula at this distance then is $7.6 \mathrm{pc}$, if it lies in the sky plane, and the separation between the northern $\left(\mathrm{N}_{1}\right)$ and southern $\left(\mathrm{N}_{2}\right)$ nucleus is about $2 \mathrm{pc}$.

We estimated the total mass of the SkN using the dust mass relation by Hildebrand (1983). Based on fluxes of $3.22 \times$ $10^{4} \mathrm{MJy} / \mathrm{str}$ at $100 \mu \mathrm{m}$, and assuming a gas-to-dust mass ratio (g:d) of $150, D=0.2 \mathrm{kpc}$, and $T_{\mathrm{d}}=27 \pm 4 \mathrm{~K}$, the total mass is $3.5 \pm 2.3 M_{\odot}$. This value is parameterized in terms of their assumed distance (in $\mathrm{kpc}$ ), temperature (in $\mathrm{K}$ ) and gas-to-dust ratio $(\mathrm{g}: \mathrm{d})$ as $3.5 \pm 2.3(\mathrm{D} / 0.2)^{2}\left(T_{\mathrm{d}} / 27 \pm 4\right)^{0.37}(\mathrm{~g}: \mathrm{d} / 150)^{1} M_{\odot}$. This value is at the lower end of the mass range of Odenwald's (1988) cometary clouds of category II (3-40 $\left.M_{\odot}\right)$.

In order to find possible candidates for the shaping of the nebula, we used SIMBAD to locate discrete sources. There are 272 entries for a $4.5^{\circ} \times 4.5^{\circ}$ region: 178 stars, 29 IRAS sources and 65 galaxies. Figure 4 shows their distribution as well as a sketch of the nebula. In general, all appear to be rather randomly distributed. There were 8 IRAS sources found in the region of the nebula, but no IRAS source is found at the maxima (nuclei) in the contour maps. 

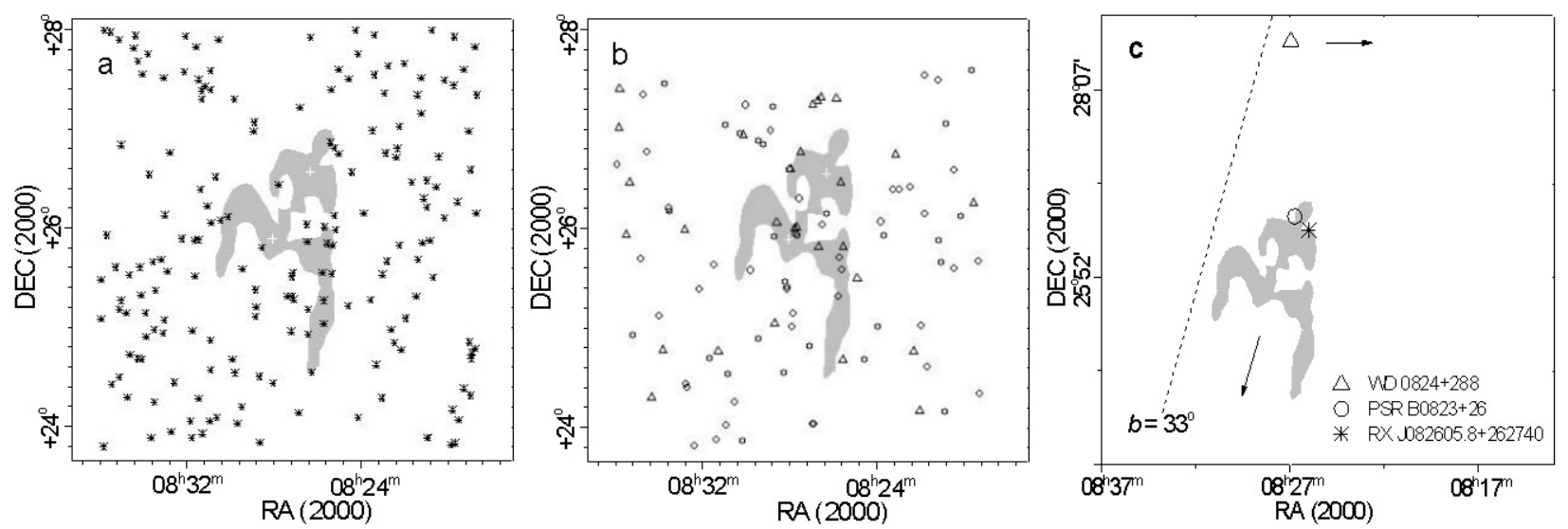

Fig. 4. Known discrete sources in the field of the nebula. a) stars $(*)$, b) galaxies (o) and IRAS point sources ( $\triangle$ ). In c) the sources RX J082605.8+262740 $(*)$, WD $0824+288(\triangle)$ and PSR B0823+26 (o) are shown. The grey structure is a sketch of the nebula as it appears at $100 \mu \mathrm{m}$. The arrows represent the p.m. of WD B0824+288 and PSR B0823+26, respectively. The dashed line in c) shows the Galactic latitude $b=+33^{\circ}$.

\section{Discrete sources in the field of the nebula}

The structure of the SkN suggests that - as one of the possibilities - a wind might blow from the north-west, leading to the cone-like morphology. To this end, we searched for any (stellar) objects which might be capable of shaping an interstellar cloud of small or moderate mass; such an object should be located at or around the head of the nebula and along the extrapolated major axis, i.e. towards the north or north-west. With SIMBAD, we found three possible candidates, namely an M-type emission star (RX J082605.8+262740), a carbon dwarf star (WD 0824+288) and a pulsar (PSR B0823+26). These were selected because (1) all of them are rather nearby, (2) they might emit a wind in the course of their evolution, (3) they show some peculiar properties, and (4) they are placed at a suitable apparent location with respect to the nebula. Their positions (and proper motions, if known) are shown in Fig. 4c.

\subsection{An M-type emission star and a dwarf carbon star}

RX J082605.8+262740 (RIXOS 206-517; RA =08 $26^{\mathrm{m}} 05^{\mathrm{s}} .8$, Dec $=+26^{\circ} 27^{\prime} 42^{\prime}(\mathrm{J} 2000)$ ) is an M-type emission star showing $\mathrm{X}$-ray features. The source was detected in the RIXOS survey (Mittaz et al. 1999), which registered objects down to a flux limit of $3 \times 10^{-14} \mathrm{erg} \mathrm{cm}^{-2} \mathrm{~s}^{-1}(0.5-2 \mathrm{keV})$. The proper motion $\left(\mu_{\alpha}, \mu_{\delta}\right)$ of this star is unknown. Its position looks promising (Fig. 4c) for shaping the nebula. We roughly estimated the distance of the star: its brightness in $B$ and $V$ is 16.5 and 15.34, respectively (Mason et al. 2000). Assuming negligible extinction we found, as a rough guess, $D=17 \mathrm{pc}$. At this distance the total mass and the physical size of the nebula would become $0.025 \pm 0.017 M_{\odot}\left(T_{\mathrm{d}}=27 \pm 4 \mathrm{~K}, \mathrm{~g}: \mathrm{d}=150\right)$ and $0.05 \mathrm{pc}$, respectively. This would make the $\mathrm{SkN}$ a very small cloudlet within the Local Bubble.

WD 0824+288 (RX J0827.0+2844; RA $=08^{\mathrm{h}} 27^{\mathrm{m}} 05^{\mathrm{s}} .1$, Dec $\left.=+28^{\circ} 44^{\prime} 02^{\prime \prime}(\mathrm{J} 2000)\right)$ is a dwarf carbon $(\mathrm{dC})$ star positioned at $\sim 2^{\circ}$ north from the northern nucleus $\left(\mathrm{N}_{1}\right)$ of the nebula (Fig. 4c). The object $\mathrm{dC}$ is hot $(\sim 52 \mathrm{kK}), \operatorname{dim}(V=$ 14.22) and has an average mass of $0.70 M_{\odot}$ (Green et al. 2000;
Green 2000; Napiwotzki et al. 1999) and proper motion $\left(\mu_{\alpha}=\right.$

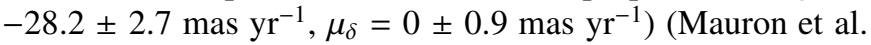
2004, private communication). Heber et al. (1993) noticed that this star shows variability due to heating effects from a hot DA companion - the reason why this dwarf is visible in the optical spectrum. In general, dwarfs are unlikely to possess active chromospheres or undergo mass loss, but WD 0824+289 is a special case, where heating of the dwarf by a hot DA companion causes the emission (Downes et al. 2004). This dwarf is called a smoking gun for the existence of dust (Green et al. 2000). We roughly estimated the distance to this dwarf using a value of the absolute magnitude estimated by Green (2000) for all $\mathrm{dCs}$, and found about $70 \mathrm{pc}$. Then, the total mass of the nebula using the same values for the dust-to-mass ratio and dust colour temperature as mentioned above is $0.43 \pm 0.29 M_{\odot}$. At $D=70 \mathrm{pc}$, the dC star would be about $2.3 \mathrm{pc}$ away from the nucleus $\mathrm{N}_{1}$ - an uncomfortably large physical separation for shaping the SkN.

PSR B0823+26 (PSR J0826+2637; RA $=08^{\mathrm{h}} 26^{\mathrm{m}} 51^{\mathrm{s}} .38$, Dec $\left.=+26^{\circ} 37^{\prime} 24^{\prime \prime}(\mathrm{J} 2000)\right)$ is a rather well studied pulsar with a proper motion $\left(\mu_{\alpha}=61 \pm 3 \operatorname{mas}_{\mathrm{yr}^{-1}}, \mu_{\delta}=-90 \pm\right.$ 2 mas $\mathrm{yr}^{-1}$ ) whose direction practically coincides with the long axis of the nebula (Fig. 4c). This pulsar has a very nearly equatorial geometry, in that both magnetic poles and the sightline all fall close to the rotational equator of the star (Rankin \& Rathnasree 1995). The transverse velocity, spin-down age and distance of PSR B0823+26 are $194 \pm 34 \mathrm{~km} \mathrm{~s}^{-1}, 4.92 \mathrm{Myr}$ and $0.36 \mathrm{kpc}$, respectively (Gwinn et al. 1986). Very recently, the $\mathrm{X}$-ray emission properties of this pulsar was studied with the XMM-Newton and it was found that the emission is largely dominated by nonthermal processes (Becker et al. 2004).

\subsection{A strange superposition}

The facts that both the position of PSR B0823+26 is at the head of the Skeleton Nebula and that the proper motion direction of the former coincides with the long axis of the latter is remarkable. Are these a chance superpositions? Unfortunately there is no convincing way to find out this, since a lot of structure in 
the IRAS maps exists at all scales, and particularly there is a multitude of variously shaped clouds, blobs, arcs, filaments, holes, etc. Nevertheless, one of us (BA) has started a study to address this problem of superpositions on IRAS maps.

To roughly estimate the probability to find such a superposition we surveyed the IRAS dust structures around all nearby ( $D \leq 1 \mathrm{kpc}$ ) pulsars using the ATNF Pulsar database (http://www . atnf.csiro.au/research/pulsar/). To this end, we used SkyView, selected the image scaling "Histogram equalization" (which permits to bring out very faint structures), used the colour table "B-W Linear" and selected $1^{\circ} \times 1^{\circ}, 5^{\circ} \times$ $5^{\circ}$ and $10^{\circ} \times 10^{\circ}$ fields, at $12,25,60$ and $100 \mu \mathrm{m}$, centered on each of the 70 nearby pulsars. We looked for all isolated emission regions of filamentary, elliptical, and circular shape. We found 5 cases in which pulsars could perhaps be associated with extended dust structures, namely PSR J2124-3358, PSR J2307+2225, PSR J1730-2304, PSR J0108-1431 and PSR J1909-3744. J2124-3358 has an associated H-alpha nebula (Gaensler et al. 2002). However, none of these dust features shows anything special (as to direction, location of the pulsar near the centre of dust cavities and/or dust enhancements etc.) Particularly, no obvious superposition with an isolated nebula, similar to the case of the SkN, could be found. Hence, we suspect that the association of PSR B0823+26 with the SkN might be real - or we are misguided by a quite improbable chance superposition. Having this strange result in mind we also looked whether there is any other pulsar in the projected neighborhood of the SkN. The closest (in projection) is PSR J0754+3231, which is $\sim 9^{\circ}$ north-west of PSR B0823+26. Its distance is $3.92 \mathrm{kpc}$ (Taylor et al. 1993), 11 times farther away than PSR B0823+26.

Is there anything that makes PSR B0823+26 a special case among pulsars? Clegg et al. (1993) found that its $1400 \mathrm{MHz}$ dynamic spectrum exhibits a sharp discontinuity. The changes occurred over time scales of $\sim 2 \mathrm{~min}$. They believe that such a discontinuity is the result of strong refraction in the interstellar medium. We may speculate: is perhaps a part of the $\mathrm{SkN}$ acting as a lens for this refraction, that is, is our nebula at $\leq 360 \mathrm{pc}$ ? To get an idea of the dimensions involved: Clegg et al. (1993) explained physical characteristics of the lens system and derived a minimum lens size of $0.1 \mathrm{AU}$ assuming that the lens is located halfway to the pulsar. It is estimated that a lens of size $\sim 0.7$ AU located halfway ( $180 \mathrm{pc}$ ) to the pulsar would have a diffractive time scale for fluctuation of approximately $2 \mathrm{~min}$.

If we assume that the $\mathrm{SkN}$ is at about the same distance as PSR B0823+26 (i.e., $360 \mathrm{pc}$ ), the total mass of the nebula would become $11.3 \pm 7.6 M_{\odot}\left(T_{\mathrm{d}}=27 \pm 4 \mathrm{~K}, \mathrm{~g}: \mathrm{d}=150\right)$ and its maximum physical size (if in the sky plane) would amount to $16.8 \mathrm{pc}$. Anyway, despite the remarkable location and proper motion direction of the pulsar and the morphology of the nebula which led us to suspect that PSR B0823+26 might possibly shape the $\mathrm{SkN}$, we have to admit that i) up to now, no interaction of this kind - namely eroding an interstellar cloud by an approaching or closely passing pulsar - has been found; ii) we would at least expect distinctly different physical conditions (heating) at the part of the cloud which is closer to the pulsar - but nothing like this can be seen in the $\mathrm{SkN}$; iii) it is hard to imagine how such a shaping by a neutron star could take place in principle. We intend to study shaping mechanisms in the future, where also the possible contribution of a pulsar nebula will be taken into account.

\section{Reynolds number hydrodynamics}

The Skeleton Nebula could be shaped via interaction with the ambient medium. Oort $(1969,1970)$ has already suggested that structures of clouds could be understood by studying their collision with the ambient ISM. During a collision, shock fronts will form that convert some of the kinetic energy of the cloud into thermal energy, heating the gas possibly to $\sim 10^{6} \mathrm{~K}$. In hydrodynamic theory, the Reynolds number $(R)$ is of relevance here, providing a dimensionless indication of whether the passes of a body through a fluid will result in turbulent motion. Using Eq. (1) given by Odenwald \& Rickard (1987), we roughly estimate the $R$ value for the $\mathrm{SkN}$ assuming that it is falling towards the Galactic plane. We emphasize that the location of the main axis of the $\mathrm{SkN}$ is almost parallel to the Galactic plane (see Fig. 4), making such an interaction not very plausible.

Let us assume a distance of 200 pc (as Odenwald 1988 did for his category II clouds) and estimate the $R$ value. The minimum value of $R$ is found to be $\sim 9$ (assuming a coefficient of viscosity for fully ionized hydrogen gas as provided by Spitzer (1962) for $T>10^{4} \mathrm{~K}$, and a grain size, density and emissivity as suggested by Hildebrand (1983) for $100 \mu \mathrm{m}$ ) for a terminal velocity (=radial velocity/sin $b$ ) of $5 \mathrm{~km} \mathrm{~s}^{-1}$. The $R$ value goes up to 90 if the terminal velocity of the nebula would be 10 times higher. This range of $R$ (i.e., 9-90) suggests that the body (i.e. our nebula) might be in the transition phase between a laminar and a turbulent flow. At this stage one can assume that vortices generate faster than that of the dissipation via molecular diffusion.

The situation will be different if our nebula is closely associated with the emission star RX J082605.8+262740. In this case, $R<1$ for a terminal velocity $<100 \mathrm{~km} \mathrm{~s}^{-1}$ (radial velocity $\sim 54 \mathrm{~km} \mathrm{~s}^{-1}$ at $32^{\circ}$ latitude), indicating a laminar flow. The $R$ value turns out to be $>100$ if the nebula is at the same distance as the pulsar PSR B0823+26. In this case, turbulence develops in the downstream.

\section{Conclusions}

A new infrared nebula $\left(\mathrm{RA}=08^{\mathrm{h}} 27^{\mathrm{m}}\right.$, Dec $\left.=+25^{\circ} 54^{\prime}(\mathrm{J} 2000)\right)$ with an apparent size of $\sim 140^{\prime} \times 70^{\prime}$ was detected on $60 \mu \mathrm{m}$ and $100 \mu \mathrm{m}$ IRAS maps. We have carried out far-IR and optical studies of this spectacular cone-like nebula. We found three discrete sources which might perhaps be responsible for shaping this nebula: the M-type emission star RX J082605.8+262740, the carbon dwarf star WD 0824+288 and the pulsar PSR B0823+26. Among them, the pulsar PSR B0823+26 appears to be the prime candidate because of its position, proper motion, distance and the presence of a discontinuity in $1400 \mathrm{MHz}$ dynamic spectrum, which suggests refraction by interstellar or ambient matter. However, how could a pulsar erode an interstellar cloud? We cannot offer any explanation and hence assume that another shaping mechanism is at work. A possible interaction of the 
nebula with the ambient ISM is discussed in terms of Reynolds number hydrodynamics. This discussion suggests that the nebula might be in a transition phase between laminar and turbulent motion. Anyway, the process or source responsible for shaping this nebula has not been found yet.

Acknowledgements. We acknowledge the unknown referees for their constructive criticism and numerous useful comments which have allowed to improve the paper. We thank the Austrian Science Fund (Fonds zur wissenschaftlichen Forschung) for support of the project P15316. B. Aryal acknowledges Tribhuvan University, Kathmandu, Nepal for granting study leave.

\section{References}

Becker, W., Weisskopf, M. C., Tennant, A. F., et al. 2004, ApJ, 615, 908

Clegg, A. W., Fiedler, R. L., \& Cordes, J. M. 1993, ApJ, 409, 691

Downes, R. A. 2004, AJ, 127, 2238

Dwek, E. 1986, ApJ, 302, 363

Gaensler, B. M., Jones, D. H., \& Stappers, B. W. 2002, ApJ, 580, L137

Green, P. J. 2000, in The Carbon Star Phenomenon, Proc. of the 177th

IAU symp., ed. R. F. Wing (Dordecht: Kluwer), 27

Green, P. J., Ali, B., \& Napiwotzki, R. 2000, ApJ, 540, 992

Gwinn, C. R., Taylor, J. H., Weisberg, J. M., \& Rawley, L. A. 1986, AJ, 91, 338
Heber, U., Bade, N., Jordan, S., \& Voges, W. 1993, A\&A, 267, L31

Henning, Th., Pfau, W., \& Altenhoff, W. J. 1990, A\&A, 227, 542

Hildebrand, R. H. 1983, Q. Jl. R. astr. Soc., 24, 267

Holmberg, E. 1946, Medd. Lund Astron. Obs. Ser. VI, No. 117

IRAS Catalogs and Atlases, Explanatory Supplement 1985, ed. C. A. Beichman, G. Neugebauer, H. J. Habing, P. E. Clegg, \& T. J. Chester (Washington: US Government Printing Office)

Mason, K. O., Carrera, F. J., Hasinger, G., et al. 2000, MNRAS, 311, 456

Mauron, N., Azzopardi, M., Gigoyan, K., \& Kendall, T. R. 2004, A\&A, 418, 77

Mebold, U., Cernicharo, J., \& Velden, L. 1985, A\&A, 151, 427

Melatos, A., \& Melrose, D. B. 1996, MNRAS, 279, 1168

Mittaz, J. P. D., Carrera, F. J., Romero-Colmenero, E., et al. 1999, MNRAS, 308, 233

Napiwotzki, R., Green, P. J., \& Saffer, R. A. 1999, ApJ, 517, 399

Odenwald, S. F. 1988, ApJ, 325, 320

Odenwald, S. F., \& Rickard, L. J. 1987, ApJ, 318, 702

Oort, J. H. 1969, Nature, 224, 1158

Oort, J. H. 1970, A\&A, 7, 381

Puget, J. L., Leger, A., \& Boulanger, F. 1985, A\&A, 142, L19

Rankin, J. M., \& Rathnasree, N. 1995, ApJ, 16, 327

Spitzer, L. 1962, Physics of Fully Ionized Gases (NY: Interscience)

Taylor, J. H., Manchester, R. N., \& Lyne, A. G. 1993, ApJS, 88, 529

Weiland, J., Blitz, L., Dwek, E., et al. 1986, ApJ, 306, L101 